\title{
Spironolactone and Colitis: Increased Mortality in Rodents and in Humans
}

\author{
Laura A. Johnson, BS, * Shail M. Govani, MD, MSc, ${ }^{*}$ Joel C. Joyce, MD, ${ }^{+}$Akbar K. Waljee, MD, MSc,* \\ Brenda W. Gillespie, PhD, ${ }^{\neq}$and Peter D.R. Higgins, MD, PhD, MSc(CRDSA)*
}

Background: Crohn's disease causes intestinal inflammation leading to intestinal fibrosis. Spironolactone is an antifibrotic medication commonly used in heart failure to reduce mortality. We examined whether spironolactone is antifibrotic in the context of intestinal inflammation.

Methods: In vitro, spironolactone repressed fibrogenesis in transforming growth factor beta (TGF- $\beta$ )-stimulated human colonic myofibroblasts. However, spironolactone therapy significantly increased mortality in two rodent models of inflammation-induced intestinal fibrosis, suggesting spironolactone could be harmful during intestinal inflammation. Since inflammatory bowel disease (IBD) patients rarely receive spironolactone therapy, we examined whether spironolactone use was associated with mortality in a common cause of inflammatory colitis, Clostridium difficile infection (CDI).

Results: Spironolactone use during CDI infection was associated with increased mortality in a retrospective cohort of 4008 inpatients (15.9\% vs. $9.1 \%, n=390$ deaths, $P<0.0001)$. In patients without liver disease, the adjusted odds ratio (OR) for inpatient mortality associated with 80 mg spironolactone was 1.99 (95\% confidence interval [CI]: 1.51-2.63) In contrast to the main effect of spironolactone mortality, multivariate modeling revealed a protective interaction between liver disease and spironolactone dose. The adjusted OR for mortality after CDI was 1.96 (95\% CI: 1.50-2.55) for patients without liver disease on spironolactone vs. 1.28 (95\% CI: 0.82-2.00) for patients with liver disease on spironolactone when compared to a reference group without liver disease or spironolactone use.

Conclusions: We propose that discontinuation of spironolactone in patients without liver disease during CDI could reduce hospital mortality by 2-fold, potentially reducing mortality from CDI by 35,000 patients annually across Europe and the U.S.

(Inflamm Bowel Dis 2012;18:1315-1324)

Key Words: spironolactone, mortality, colitis, Crohn's disease, Clostridium difficile

$\mathrm{T}$ he renin-angiotensin-aldosterone system (RAAS) has been implicated in fibrosis of multiple organs including heart, liver, pancreas, and kidney. ${ }^{1-3}$ Spironolactone (SPIR), a competitive aldosterone receptor antagonist, is a potent antifibrotic, improves the survival of congestive

Additional Supporting Information may be found in the online version of this article.

Received for publication September 13, 2011; Accepted September 28, 2011.

From the *University of Michigan, Department of Internal Medicine, Ann Arbor, Michigan, 'Medical College of Wisconsin, Department of Dermatology, Milwaukee, Wisconsin, ${ }^{\star}$ University of Michigan, Department of Biostatistics, School of Public Health, Ann Arbor, Michigan.

Reprints: Peter D.R. Higgins, MD, PhD, MSc(CRDSA), Assistant Professor in Gastroenterology, Division of Gastroenterology and Hepatology, Department of Internal Medicine, University of Michigan Medical Center, SPC 5682, Room 6510D, Medical Science Research Building One, 1150 West Medical Center Dr., Ann Arbor, MI 48109-0682 (e-mail: phiggins@med.umich.edu).

Funded by NIH grant K08DK080172 (to P.D.R.H.) and National Center for Research Resources (NCRR) grant UL1RR024986 (to A.K.W.).

Copyright (C) 2011 Crohn's \& Colitis Foundation of America, Inc. DOI 10.1002/ibd.21929

Published online 13 November 2011 in Wiley Online Library (wileyonlinelibrary.com). heart failure patients, and is protective in several rodent models of organ fibrosis. ${ }^{4-7}$

Crohn's disease (CD) frequently produces intestinal fibrosis and strictures requiring surgical intervention., ${ }^{8,9}$ Current therapies control inflammation and improve symptoms, yet do not alter the development of intestinal fibrosis and the natural history of $\mathrm{CD} .^{10}$ Intestinal wound healing is mediated by myofibroblasts, which are postulated to be the major contributors to intestinal fibrosis. ${ }^{11}$ Myofibroblasts are characterized by expression of $\alpha$-smooth muscle actin $(\alpha \mathrm{SMA})$ and are activated by transforming growth factorbeta1 (TGF- $\beta$ ) in fibrotic diseases. ${ }^{12}$ Angiotensin II stimulates TGF- $\beta$ in cardiac myofibroblasts, therefore we hypothesized that downstream aldosterone blockade of this signaling should prevent TGF- $\beta$-induced fibrosis in vitro and in vivo.

We investigated whether SPIR reduces intestinal fibrosis in an in vitro colonic myofibroblast model and in two rodent models of intestinal fibrosis. We determined that SPIR is antifibrotic in vitro. Paradoxically, in two different rodent colitis models, SPIR therapy during intestinal inflammation produced rapid and significant mortality. To 
evaluate the clinical relevance of our rodent mortality results, we considered evaluating the effect of SPIR on mortality of inflammatory bowel disease (IBD) patients with active inflammation, but SPIR use is rare in patients with IBD. As an alternative, we examined the mortality associated with SPIR use in an easily identifiable and common source of human colonic inflammation, Clostridium difficile infection (CDI). We performed a multivariate logistic regression in a retrospective cohort of inpatients with CDI to evaluate the effect of SPIR use on CDI mortality.

\section{MATERIALS AND METHODS}

\section{In Vitro Model Reagents}

Human recombinant TGF- $\beta 1$ was obtained from R\&D Systems (Minneapolis, MN). SPIR and canrenoic acid were purchased from Sigma Aldrich (St. Louis, MO). Enalprilat was acquired from Hopsira (Lake Forest, IL). Eplereone was purchased from Tocris (Ellisville, MO). Losartan was obtained from Merck (Whitehouse Station, NJ), and aliskiren from Novartis (East Hanover, NJ).

\section{In Vitro Myofibroblast Culture Methods}

Early passage (3-12) colonic human fibroblast CCD18Co cells (CRL-1459 from American Type Culture Collection [ATCC], Manassas, VA) were cultured in alpha-MEM (Invitrogen, Carlsbad, CA) supplemented with $10 \%$ fetal bovine serum and subcultured weekly. For in vitro experiments, cells were plated at $30 \%-40 \%$ confluence and serum-starved prior to treatment with TGF- $\beta$ and other compounds. To stimulate a fibrotic phenotype, CCD-18co cells at $30 \%-40 \%$ confluence were serum-starved for 24 hours prior to treatment with $1 \mathrm{ng} / \mathrm{mL}$ TGF- $\beta$ or $1 \mathrm{ng} / \mathrm{mL}$ TGF- $\beta$ and $100 \mu \mathrm{M}$ SPIR or $1 \mathrm{mM}$ canrenone for 48 hours. In the RAAS inhibitor experiments, CCD-18co cells were stimulated with $1 \mathrm{ng} / \mathrm{mL}$ TGF- $\beta$ to which either $50 \mu \mathrm{M}$ aliskiren, $100 \mathrm{nM}$ enalprilat, $10 \mu \mathrm{M}$ losartan, or $50 \mu \mathrm{M}$ eplerenone was added. Cells were harvested after 48 hours.

\section{Protein Expression}

Total cellular lysates were subjected to sodium dodecyl sulfate-polyacrylamide gel electrophoresis (SDS-PAGE) as previously described. ${ }^{13}$ $\alpha$ SMA protein was detected with a mouse monoclonal antibody to human $\alpha$ SMA protein (Sigma). GAPDH protein expression was used as a loading control using a mouse antibody to GAPDH (Chemicon, Temecula, CA). After application and washing of the primary antibodies, membranes were incubated with a horseradish peroxidase (HRP)-conjugated antibody to mouse immunoglobulin $\mathrm{G}$ ( $\mathrm{IgG}$ ) (Invitrogen, Carlsbad, CA) and developed using the Pierce Dura detection system (Rockford, IL). The resulting autoradiographs were scanned and the images were quantitated using the ImageJ analysis software (available at http://rsbweb. nih.gov/ij/).

\section{Gene Expression Studies}

RNA from CCD-18co cells was extracted using the RNeasy kit (Qiagen, Valencia, CA). cDNA was generated by reverse transcription of $2 \mu \mathrm{g}$ of total RNA using the Superscript First Strand RT kit (Invitrogen). Quantitative real-time polymerase chain reaction (PCR) was performed for Acta2, Collal, Ctgf, and Gapdh with the TaqMan gene expression assays (ABI, Foster City, CA) on a Stratagene Mx3000P realtime PCR system (La Jolla, CA). Gene expression was normalized to Gapdh as the endogenous control and fold-changes (RQ) relative to uninfected controls (no Tx) were calculated using the $\Delta \Delta \mathrm{Ct}$-method. ${ }^{14}$

\section{Rodent Models}

Chronic colitis and subsequent fibrosis was induced using the rat trinitrobenzene sulfonic acid (TNBS) enema model as previously described. ${ }^{15}$ Then $0.5,2.5,10$, or $20 \mathrm{mg} /$ $\mathrm{kg}$ /day SPIR was administered twice daily by oral gavage. Control animals received vehicle twice daily orally.

In the mouse colitis and intestinal fibrosis model, mice were infected with Salmonella typhimurium as previously described. ${ }^{16}$ Briefly, female 8-12-week-old 129S1/SvImJ mice (Jackson Laboratories, Bar Harbor, ME) were divided into two groups. Half the animals received regular rodent chow, the other half received a custom rodent chow containing $2.78 \mathrm{~g}$ SPIR per kg (Harlan Teklad, Madison, WI). After 1 week, half of the animals in either the standard chow or SPIR-supplemented chow groups received $20 \mathrm{mg}$ of streptomycin in 0.1 M Hank's balanced salt solution (HBSS) by oral gavage 24 hours prior to oral infection with $3 \times 10^{6}$ colony-forming units (CFU) of S. typhimurium strain SL1344 in $100 \mu \mathrm{L} 0.1 \mathrm{M}$ HEPES $(\mathrm{pH}=8.0)$. Uninfected animals received streptomycin and HEPES on the same schedule as the infected groups. Given the $100 \%$ mortality in the S. typhimurium/SPIR group, the uninfected SPIR group was subsequently challenged at day 16 of the 21-day experiment with $S$. typhimurium as described above. Surviving mice were euthanized at 21 days post- $S$. typhimurium infection. Mice were monitored for weight loss/ gain, general appearance, and health. All animal experiments were conducted with the approval and oversight of the University of Michigan UCUCA (University Committee on Use and Care of Animals).

\section{Retrospective C. difficile Colitis Study Design}

A retrospective cohort of inpatients with a discharge diagnosis of CDI at the University of Michigan Hospital, Ann Arbor, MI was reviewed with Institutional Review Board approval. The consent of participants to review their medical records was waived for this protocol, as the large number of subjects made consent impractical and the risks to subjects were minimal. The electronic medical system was queried for 


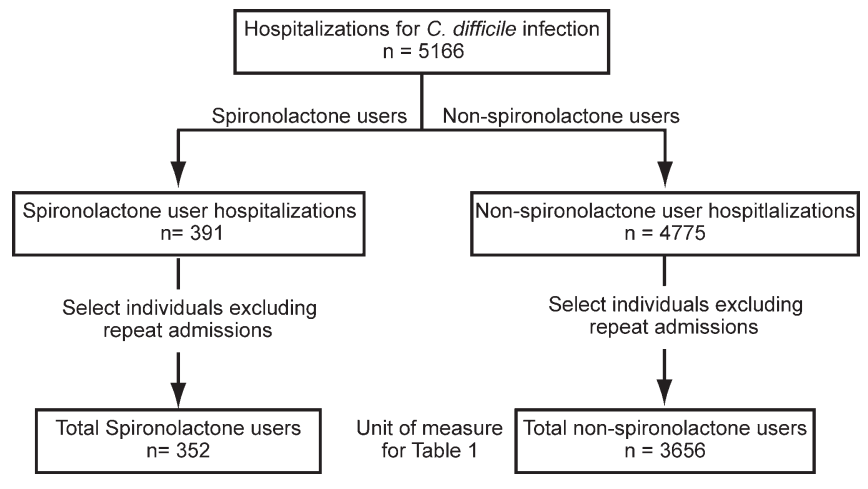

FIGURE 1. Consolidated Standards of Reporting Trials (CONSORT) flow diagram. Selection of inpatient subjects for modeling among patients with a discharge diagnosis of CDI. Readmissions for recurrent $C D I$ within 30 days of discharge were treated as a single admission for this analysis.

all inpatients older than 18 years of age with a discharge diagnosis of CDI from January 1, 2000 through December 31, 2009. Demographics, labs, discharge diagnoses, and other pertinent data were abstracted electronically. To determine SPIR dosing, a manual chart review was performed, with the highest in-hospital dose used for modeling. Patients on eplerenone therapy were excluded due to small sample size $(n=4)$. Definitions of heart failure and liver disease as well as descriptions of methods of laboratory value abstraction are detailed in the Supporting Methods. Selection of the patient sample population for modeling is detailed in Figure 1.

\section{Statistical Methods}

The descriptive statistics are reported as means \pm standard deviation (SD) or percentages. Comparisons between the SPIR users and nonusers were made by Student's $t$-test for quantitative variables and chi-square tests for categorical variables. Single predictor odds ratios (ORs) were calculated for inpatient mortality using the LOGISTIC procedure in SAS 9.2 (Cary, NC). A multivariate model of inpatient mortality was chosen using the best subset selection method in SAS while maintaining SPIR dose, liver disease, and heart failure in the model. As $18.6 \%$ of patients were hospitalized more than once, the effect of hospitalization on mortality was investigated and mortality was found to be greater in the first two hospitalizations. Therefore, the single predictor and multivariate models were adjusted for the hospitalization number for each individual, dichotomized as $\leq 2$ vs. $>2$ hospitalizations. All possible two-way interactions were tested. $P$-values less than 0.05 were considered statistically significant. All tests are two-sided.

The original Model for Endstage Liver Disease (MELD) formula, which allows for negative values, was used. ${ }^{17}$ The optimal functional form of SPIR dose in the multivariate model was assessed by comparing linear to both logarithmic and quadratic transformations using Akaike information criteria (AIC) and likelihood ratio testing. Sensitivity of the model to high doses of SPIR ( $>100 \mathrm{mg}$ ) was tested by comparing parameter estimates in a model with and without these higher doses. As stated in the Results, this did not change the significance of any of the parameters.

The logistic regression analysis strategy was based on a model for discrete survival. In our case, the time axis was hospitalization number, with death (yes/no) evaluated for each hospitalization. In such discrete survival models, time is entered as a model term. After considering continuous (linear) versus categorical variables for hospitalization number, we determined that using categories $(\leq 2$ hospitalizations vs. $>2$ ) provided the best fit. To test the robustness of our modeling method we also performed logistic regression on a random hospitalization for each patient. The results of this alternative modeling method did not change the significance of any of the parameters.

\section{RESULTS}

\section{In Vitro Studies}

To determine whether SPIR is antifibrotic in vitro, human colonic myofibroblasts (CCD-18co) were stimulated with TGF- $\beta$ to induce a fibrotic phenotype characterized by increased actin stress fiber accumulation, $\alpha \mathrm{SMA}$ protein expression, and increased expression of profibrotic genes including Acta2, Collal, and Ctgf (data not shown). SPIR repressed TGF- $\beta$ induction of $\alpha$ SMA protein expression in a dose-dependent manner (Fig. 2A). SPIR repressed actin stress fiber formation (data not shown). Both SPIR and its active metabolite, canrenone, dramatically repressed TGF$\beta$-induced $\alpha \mathrm{SMA}$ protein expression (data not shown). SPIR repressed the expression of fibrotic genes Acta2, Collal, and Ctgf to levels indistinguishable from untreated controls (Fig. 2B). Canrenone significantly repressed Acta2 mRNA expression, but its reduction in Collal and Ctgf mRNA expression were not statistically significant (Fig. 2B).

Given the antifibrotic effect of SPIR, we investigated whether blocking upstream components of the RAAS pathway (Fig. 2C) would block fibrosis in vitro. In TGF- $\beta$-stimulated myofibroblasts, other RAAS inhibitors aliskiren (renin inhibitor), enalaprilat (ACE inhibitor), and losartan (angiotensin II type I receptor blocker [ARB]) partially repressed profibrotic gene expression (Fig. 2D,E), suggesting a role for the RAAS pathway in intestinal fibrosis.

\section{Rodent Colitis Studies}

Unexpectedly, in two animal models of intestinal inflammation and fibrosis, treatment with SPIR caused significant and rapid mortality. In the rat TNBS chronic colitis model, $20 \mathrm{mg} / \mathrm{kg} /$ day SPIR (10-fold lower than well-tolerated doses in other rat models ${ }^{18}$ ) produced $67 \%$ mortality (95\% confidence interval [CI]: 0.046-0.68) (Fig. 3A). A dose-response experiment in the rat TNBS model 

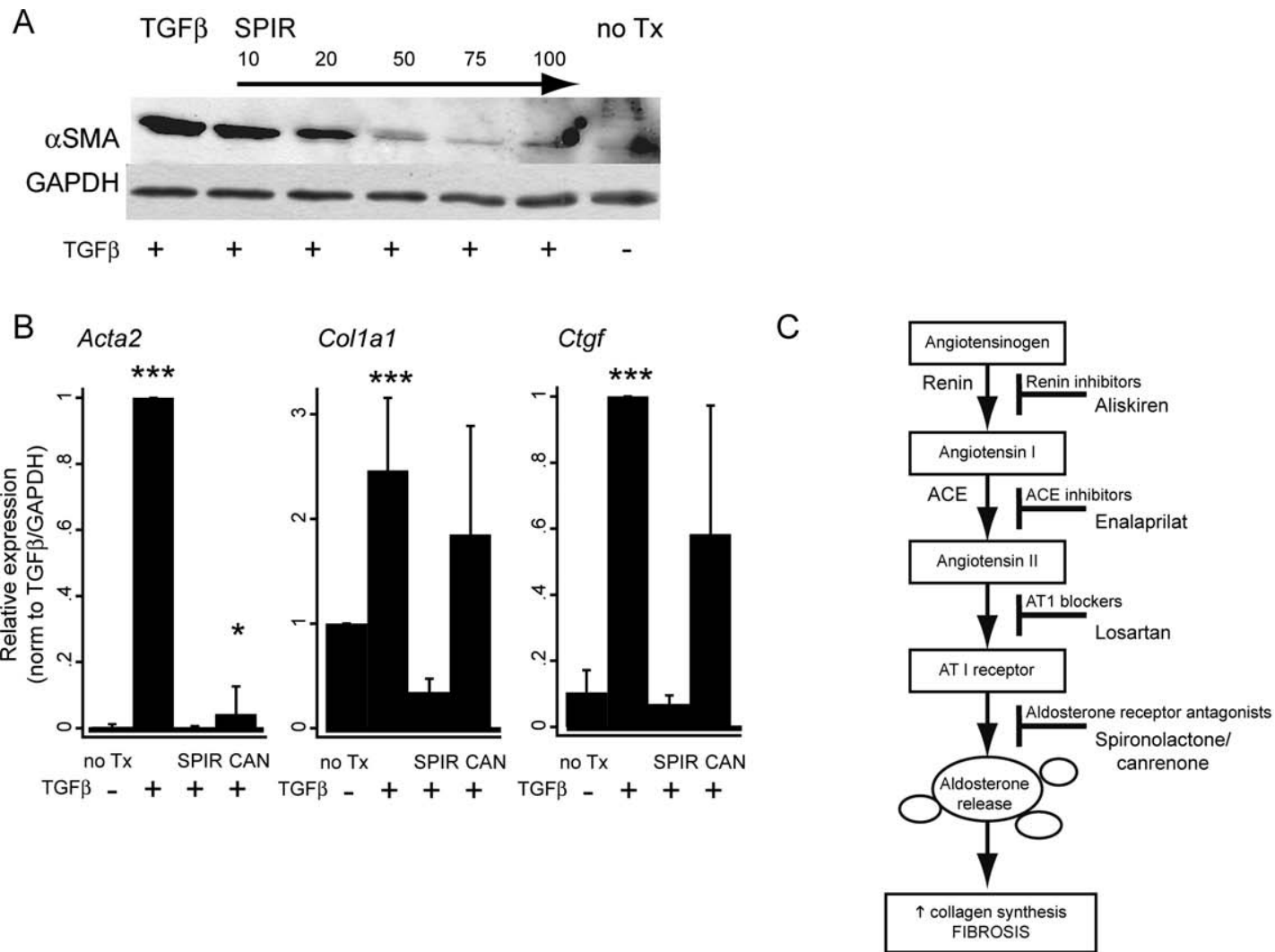

D Acta2 mRNA Expression

E
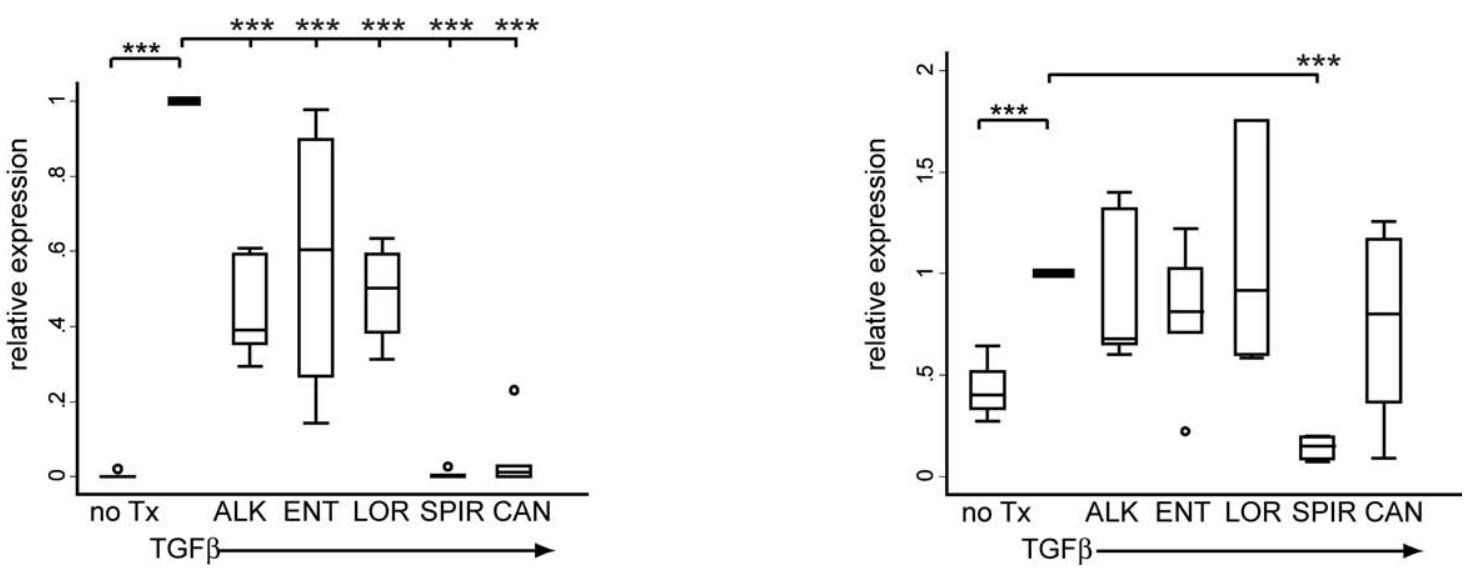

FIGURE 2. Results of in vitro studies. (A) SPIR inhibits TGF- $\beta$ induction of $\alpha$ SMA protein expression in colonic myofibroblasts. A representative western blot of $\alpha$ SMA expression in protein extracts from CCD-18co colonic myofibroblasts stimulated for 24 hours with TGF- $\beta$ is shown. Increasing amounts of SPIR from $10 \mu \mathrm{M}$ to $100 \mu \mathrm{M}$ reduce $\alpha$ SMA expression to levels comparable to unstimulated cells (no Tx). GAPDH expression serves as the loading control for the amount of protein. (B) Treatment of TGF- $\beta$ stimulated CCD-18co cells with SPIR represses expression of Acta2, Col1a1, and Ctgf. A metabolite of SPIR, canrenone (CAN), partially represses profibrotic gene expression. (C) The role of the RAAS pathway in fibrosis and the relationship of pathway inhibitors. (D) Inhibitors of the RAAS pathway aliskiren (ALK), enalaprilat (ENT), and losartan (LOR) partially repress TGF- $\beta$ induction of fibrotic genes with partial repression of Acta2 expression but have minimal effect on Col1a1 expression (E). Results are from nine independent experiments. Asterisks denote statistically significant comparisons between untreated control cells (no Tx) and the treatment groups. Brackets denote comparisons between TGF- $\beta$ treated and other treatment groups. ${ }^{*} P<0.05,{ }^{* *} P<0.001$.

demonstrated increased survival with decreasing SPIR dose. Mortality was $33 \%$ at $10 \mathrm{mg} / \mathrm{kg} / \mathrm{day}$, and $0 \%$ at 2.5 or $0.5 \mathrm{mg} / \mathrm{kg} /$ day (Fig. 3B). However, lower doses of SPIR did not reduce the development of fibrosis, as determined by gross pathology, histopathology, fibrotic gene expression, and protein expression ( $\alpha \mathrm{SMA}$ ) (data not shown). 

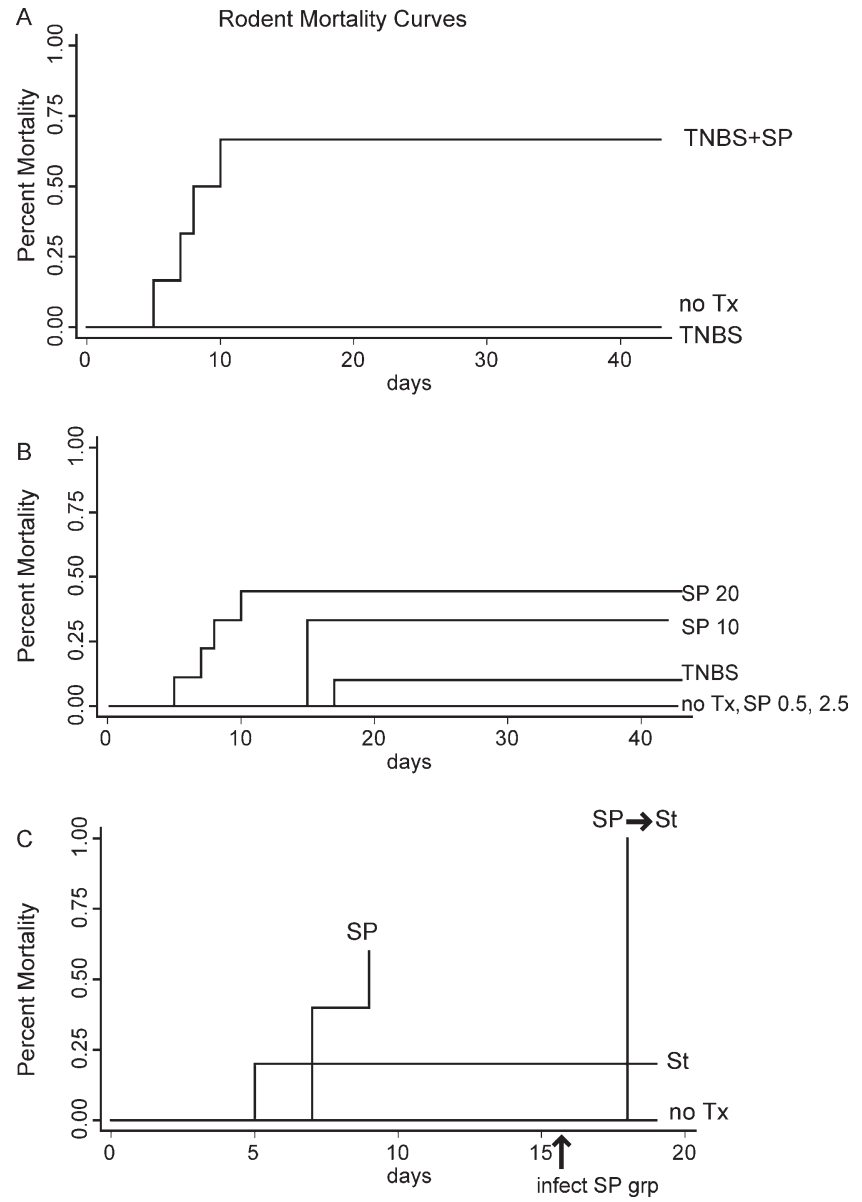

FIGURE 3. Results of rodent colitis studies. (A) Increased mortality in rats with chronic TNBS colitis treated with SPIR. Kaplan-Meier mortality estimates of rats with chronic TNBS and $20 \mathrm{mg} / \mathrm{kg} /$ day SPIR (TNBS+SP) compared to no mortality with TNBS alone and in untreated rats (no Tx). Data are from three rats per experimental group. (B) Increased mortality in rats with chronic TNBS colitis treated with SPIR is dose-dependent. The Kaplan-Meier curve demonstrates increased mortality in rats with chronic TNBS colitis treated with doses of 10 or $20 \mathrm{mg} / \mathrm{kg} /$ day SPIR (T+SP 20, T+SP 10) compared to low doses of SPIR (T+SP 0.5, T+SP 2.5), TNBS alone, or rats with no treatment (no Tx). Data are from three rats per experimental group. (C) SPIR treatment increases mortality in the $S$. typhimurium mouse model of colitis. Mortality occurred in mice with S. typhimurium-induced colitis treated with $0.7 \mathrm{mg} / \mathrm{kg} /$ day SPIR (SP+St), compared with mice infected with $S$. typhimurium without SPIR (St), uninfected mice (no Tx), or mice receiving 0.7 $\mathrm{mg} / \mathrm{kg} /$ day SPIR alone (SP). Uninfected mice which received SPIR treatment over 15 days had $0 \%$ mortality until subsequent S. typhimurium induction of colitis (SP $\rightarrow \mathrm{SP}+\mathrm{St}$ ) at day 16 (vertical arrow), which produced $100 \%$ mortality by day 5 postinfection. Data are from five mice per experimental group.

Similar experiments with losartan resulted in no mortality and no improvement in fibrosis (data not shown).

In the mouse $S$. typhimurium infection model of intestinal fibrosis, treatment with $0.7 \mathrm{mg} / \mathrm{kg} /$ day of SPIR produced $80 \%$ mortality (95\% CI: $0.69-1.00)$ by day 9 of colitis compared to $20 \%$ mortality in the S. typhimurium-infected group (Fig. 3C). No mortality occurred in uninfected mice receiving $0.7 \mathrm{mg} / \mathrm{kg} / \mathrm{day}$ of SPIR. However, when the SPIR-treated cohort was later challenged with S. typhimurium, 100\% mortality occurred by day 5 postinfection.

\section{Retrospective Study of C. difficile Colitis Clinical Outcomes}

To determine whether SPIR use was associated with increased mortality in humans with intestinal inflammation, we identified a total of 4008 inpatients with a discharge diagnosis of CDI in University of Michigan Hospital admissions from January 1, 2000 through December 31, 2009. These 4008 patients accounted for a total of 5166 CDIassociated hospitalizations in that 10 -year period. There were 352 patients on SPIR during at least one hospitalization; these patients were hospitalized 391 times. The average time between hospitalizations for SPIR users was not significantly different from nonusers, while the average number of hospitalizations for the two groups did differ (1.4 vs. $1.3, P=0.02, n=4,008$ ) (Table 1 ). In patients on SPIR therapy, the average dose was $79.9 \mathrm{mg}( \pm 71.5)$. The average age, gender ratio, and race did not differ between SPIR users and nonusers.

As expected, the group on SPIR therapy had significantly more patients with a diagnosis of heart failure $(38.9 \%$ vs. $15.0 \%, P<0.0001)$ and liver disease $(42.9 \%$ vs. $7.5 \%, P<0.0001)$ compared to nonusers of SPIR. The mortality in those patients with heart failure was 77 of 686 $(11.2 \%)$, in those with liver disease was 71 of 424 $(16.7 \%)$, and in patients with neither heart failure nor liver disease was 242 of 2898 (8.4\%). The majority of patients taking SPIR without a history of heart failure or liver disease were doing so due to hypertension or evidence of edema.

Laboratory values affected by heart or liver dysfunction (creatinine, sodium, international normalized ratio [INR], total bilirubin, potassium, albumin) were significantly different between users and nonusers of SPIR (Table 1 ), and were used as proxies for disease severity in a predictive model. Bilirubin and INR were higher in the SPIR group while sodium, albumin, and creatinine were lower. Discharge day potassium was higher in the SPIR group. The MELD ${ }^{17}$ score showed a significant difference between the two groups, while there was no significant difference in Charlson-Deyo comorbidity index. ${ }^{19,20}$ The clinical outcomes in the two groups demonstrated significant differences, with longer length of stay, higher mortality, and higher costs per hospitalization in the SPIR users.

Figure 4 shows the single predictor ORs for inpatient mortality in all patients after adjustment for hospitalization number. In this analysis $(n=5166$, patients on SPIR had 1.84 [95\% CI: 1.34-2.53] times the odds of inpatient mortality of those not on the medication). For each additional 
TABLE 1. Characteristics of Patients with C. difficile Colitis on Spironolactone Therapy

\begin{tabular}{|c|c|c|c|}
\hline Characteristic & $\begin{array}{c}\text { Spironolactone Therapy } \\
(N=352) \text { Mean }( \pm \mathrm{SD}) \text {, } \\
\text { Mean [Range] or } n(\%)\end{array}$ & $\begin{array}{l}\text { No Spironolactone } \\
\text { Therapy }(N=3656) \\
\text { Mean }( \pm \mathrm{SD}), \text { Mean } \\
{[\text { Range] or } n(\%)}\end{array}$ & $P$ value $(N)$ \\
\hline Age (years) & $57.1( \pm 14.2)$ & $57.6( \pm 17.9)$ & 0.53 \\
\hline Male & $181(51.4 \%)$ & $1859(50.9 \%)$ & 0.84 \\
\hline Race & & & 0.29 \\
\hline Caucasian & $282(80.1 \%)$ & $3025(82.7 \%)$ & \\
\hline African-American & $37(10.5 \%)$ & $369(10.1 \%)$ & \\
\hline Other & $33(9.4 \%)$ & $262(7.2 \%)$ & \\
\hline Hospitalizations (individual) & 391 & 4775 & \\
\hline No. of hospitalizations per patient & $1.4[1-6]$ & $1.3[1-12]$ & 0.02 \\
\hline Time between hospitalizations (days) & $132.5[1-1483]$ & $129.2\left[\begin{array}{ll}1 & -2383\end{array}\right]$ & 0.90 \\
\hline Heart failure & $137(38.9 \%)$ & $549(15.0 \%)$ & $<0.0001$ \\
\hline Liver disease & $151(42.9 \%)$ & $273(7.5 \%)$ & $<0.0001$ \\
\hline Diabetes & $90(25.6 \%)$ & $835(22.8 \%)$ & 0.25 \\
\hline \multicolumn{4}{|l|}{ Baseline measures (per hospitalization) } \\
\hline Sodium (mmol/L) & $134.9( \pm 5.6)$ & $137.4( \pm 4.8)$ & $<0.0001(5121)$ \\
\hline Potassium (mmol/L) & $4.2( \pm 0.6)$ & $4.1( \pm 0.5)$ & $0.0004(4582)$ \\
\hline Creatinine (mg/dL) & $1.3( \pm 0.7)$ & $1.5( \pm 1.6)$ & $<0.0001(5118)$ \\
\hline BUN (mg/dL) & $27.5( \pm 18.3)$ & $25.3( \pm 20.4)$ & $0.03(5119)$ \\
\hline INR & $1.6( \pm 1.0)$ & $1.4( \pm 1.0)$ & $0.007(3763)$ \\
\hline Albumin (g/dL) & $3.1( \pm 0.7)$ & $3.1( \pm 0.7)$ & $<0.0001(4432)$ \\
\hline Total bilirubin (mg/dL) & $3.1( \pm 5.3)$ & $1.3( \pm 3.5)$ & $<0.0001(4479)$ \\
\hline MELD & $6.6( \pm 9.0)$ & $2.5( \pm 10.1)$ & $<0.0001(3416)$ \\
\hline Charlson-Deyo Comorbidity Index & $4.1( \pm 2.7)$ & $3.9( \pm 2.7)$ & $0.25(3741)$ \\
\hline \multicolumn{4}{|l|}{ Outcomes } \\
\hline Mortality (by individual) & $56(15.9 \%)$ & $334(9.1 \%)$ & $<0.0001$ \\
\hline Mortality (by hospitalization) & $49(12.5 \%)$ & $341(7.1 \%)$ & 0.0001 \\
\hline Length of each hospitalization (days) & $25.0[1-171]$ & $17.1[1-851]$ & $<0.0001$ \\
\hline Cost per hospitalization (in $\$ 1000$ ’s) & $197.9[0-2489.4]$ & $110.4[0-6388.0]$ & $<0.0001$ \\
\hline
\end{tabular}

$25 \mathrm{mg}$ of SPIR dose, the OR for mortality was increased by $13 \%(95 \%$ CI: 6\%-20\%). Liver disease, higher MELD scores, and heart failure were associated with higher inpatient mortality, while Charlson-Deyo scores were not. ACE-I (angiotensin converting enzyme inhibitor) and ARB use were both associated with decreased mortality.

These predictors were combined in a multivariate logistic regression model $(n=4415)$ to predict inpatient mortality (Fig. 5A). We found that SPIR use remained a significant predictor of mortality, and found a statistically significant interaction between SPIR dose and liver disease, described below. Testing the other univariate predictors, we found that blood urea nitrogen (BUN), total bilirubin, albumin, and use of ACE-I and ARB medications were significant contributors to our model of inpatient mortality. No significant interaction between heart failure and SPIR use was found. In all, 4415 hospitalizations without missing values were included in this model. Hospitalization number was also included for each individual in the model; patients were more likely to die during one of the first two hospitalizations than in subsequent hospitalizations (OR 1.52 , 95\% CI: $0.92-2.50, P=0.10$ ). This model had fairly high explanatory power, with a $c$ statistic of 0.71 . The linear form of SPIR dosing in the model was statistically superior to a quadratic or logarithmic transformation. The exclusion of high doses of SPIR $(>100 \mathrm{mg})$ did not substantially alter the model.

Because of the interaction between liver disease and SPIR, patients with liver disease had a (nonsignificant) protective effect from SPIR of 29\% (OR 0.71, 95\% CI: $0.47-$ 1.07), while patients without liver disease had significantly worse outcomes with SPIR. Compared to the reference group (patients without liver disease not taking SPIR), patients with liver disease taking the average dose of SPIR 


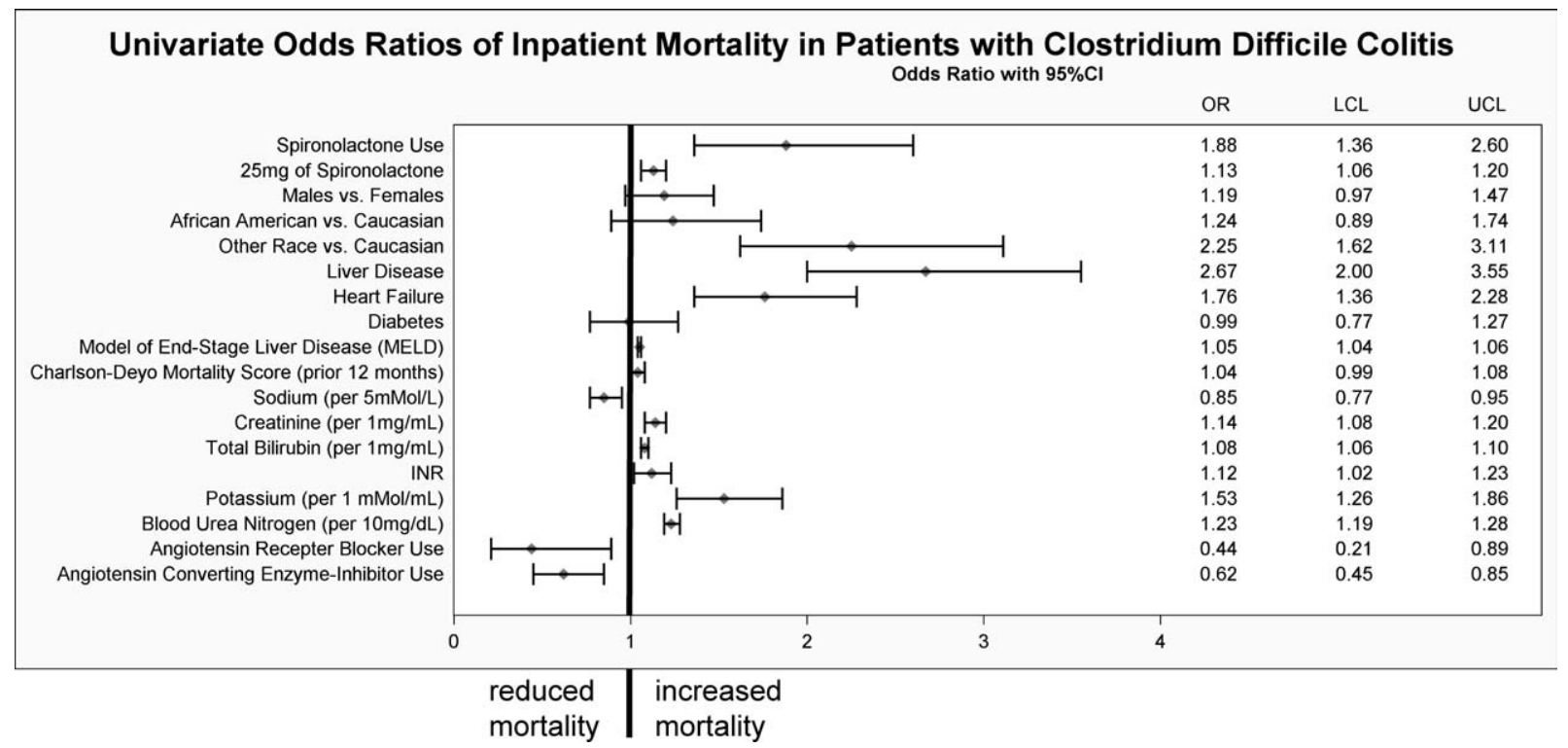

FIGURE 4. Single predictor odds ratios for inpatient mortality in patients with $C$. difficile colitis. For each predictor, the odds ratio from a logistic model for inpatient mortality was adjusted for hospitalization number ( $<2$ vs. $>2)$ and is presented with $95 \%$ Cls. A vertical dashed line at 1 represents the point of no effect.

(80 mg) had an adjusted OR for inpatient mortality of 1.07 (95\% CI: 0.70-1.64). In patients without liver disease, the adjusted OR for inpatient mortality associated with $80 \mathrm{mg}$ SPIR was 1.99 (95\% CI: 1.51-2.63) (Fig. 5A) compared to the reference group. We show the direct effects of this interaction term on mortality in Figure $5 \mathrm{~B}$, where the probabilities of inpatient mortality in patients with liver disease, heart failure, and with neither of these comorbidities are illustrated. To show the dose-response effect, we modeled the predicted probability of inpatient mortality for each disease state for patients on high-dose SPIR, low-dose SPIR, and those not taking the drug. Predicted probabilities are based on the average values of total bilirubin, albumin, and BUN, ACE-I/ARB use, and hospitalization number $\leq 2$. Patients with liver disease had a lower probability of inpatient mortality if they were using SPIR. In contrast, in patients with heart failure, who are typically on lower doses of SPIR, we demonstrate an increase in inpatient mortality with SPIR use. The patients without liver disease or heart failure were on higher average doses of SPIR, and this is reflected in a significantly increased risk of inpatient mortality.

\section{DISCUSSION}

The antifibrotic effects of aldosterone antagonists have been demonstrated in multiple organs. Clinically, the use of SPIR has a significant survival benefit in heart failure, but the effect of SPIR on intestinal fibrosis is unknown. In this study we found that SPIR represses fibrotic protein expression and the expression of profibrotic genes in intestinal myofibroblasts. However, we found that
SPIR intervention during inflammatory colitis significantly increased mortality in two rodent models of inflammatory intestinal fibrosis. We extended this finding to patients in a retrospective cohort study of clinical outcomes at a large tertiary center and found that SPIR use in patients with CDI was associated with an increased mortality rate in patients without liver disease, even after adjusting for comorbidities and disease severity.

SPIR is absorbed through the gut mucosa and metabolized in the liver. While the pharmacokinetics of SPIR metabolism are complex due to the large number of active metabolites generated, in patients with liver disease impaired SPIR metabolism has been reported. ${ }^{21-23}$ Altered hepatic SPIR metabolism may account for the differential mortality outcomes of liver disease compared to nonliver disease patients.

Our final model includes high blood urea nitrogen as a predictor of mortality, which may be a marker of dehydration and therefore severity of CDI. Based on our in vitro results, we would anticipate that $\mathrm{ACE}$ inhibitors and ARBs, as inhibitors of the RAAS, would have effects similar to SPIR, but this was not the case. The protective effect of ACE inhibitors and ARBs suggests that SPIR may have a distinct mechanism that is associated with mortality in the setting of CDI. Our findings are supported by a recent retrospective study which demonstrated ACEI/ ARB use during CDI was associated with decreased mortality. ${ }^{24}$

While we cannot infer causality with a retrospective study, several aspects of our study provide compelling 


\section{A Adjusted Odds Ratios for Inpatient Mortality in Patients with C.difficile Colitis}

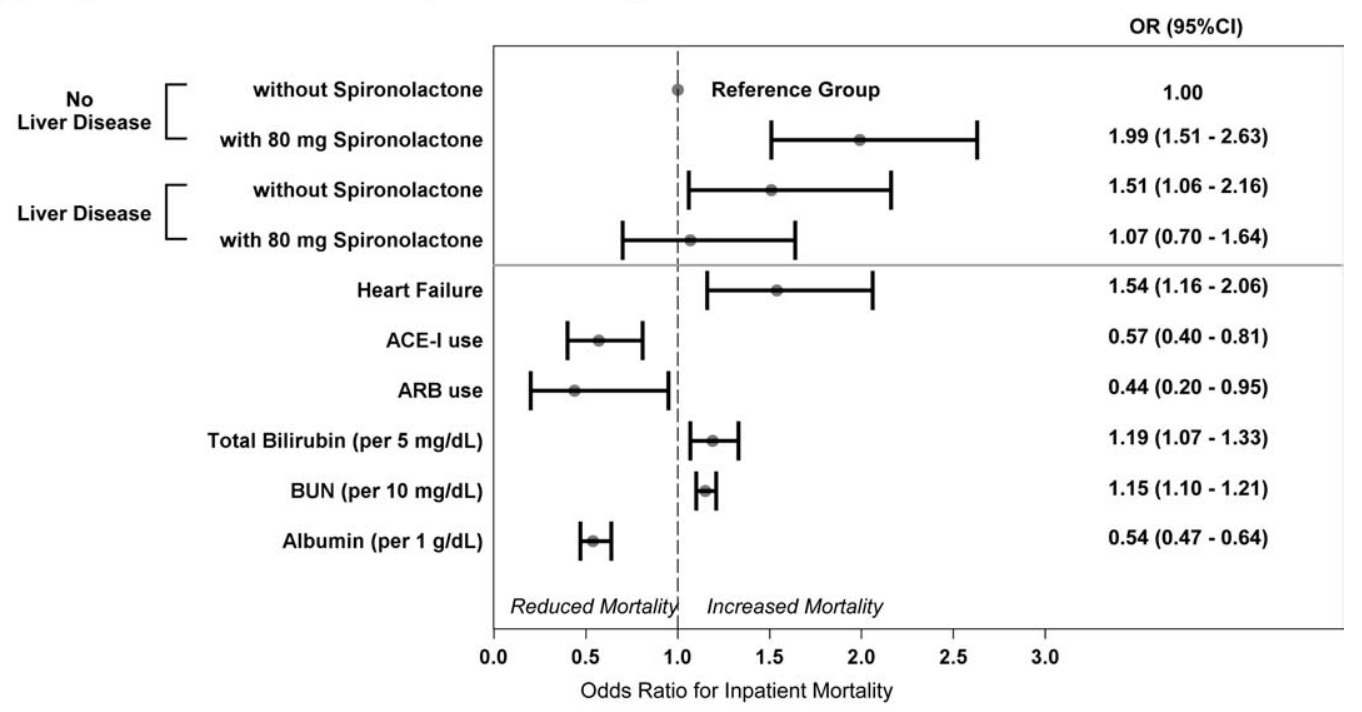

\section{B Predicted Probability of Inpaitient Mortality and Comorbidities}

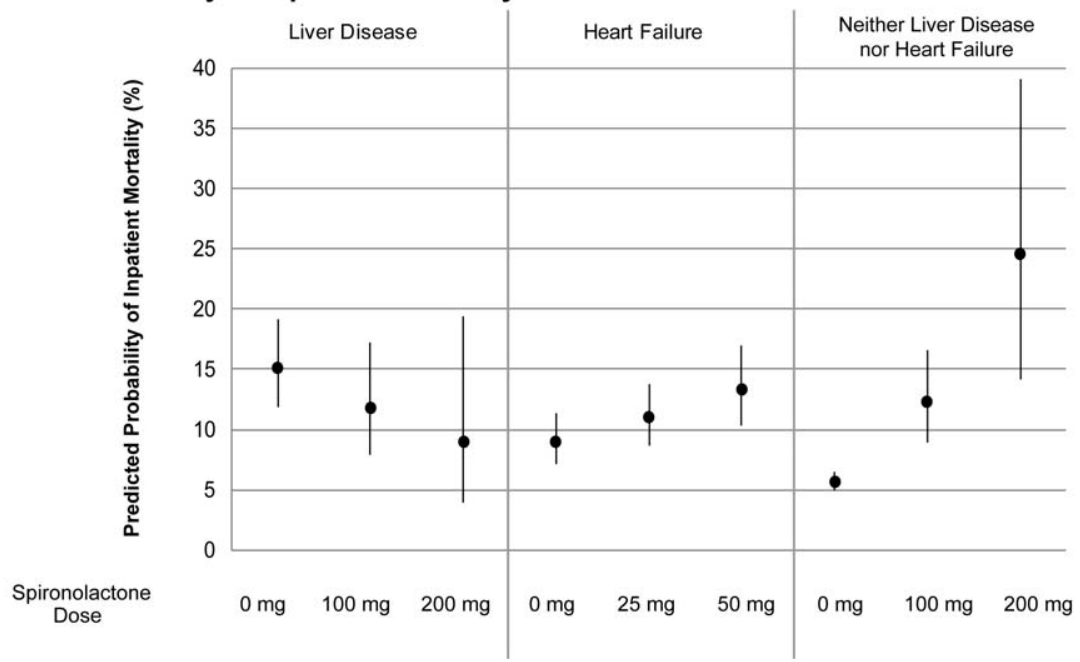

FIGURE 5. Adjusted odds ratios for and predicted probabilities of inpatient mortality in patients with $C$. difficile colitis. (A) The adjusted ORs for inpatient mortality in a multivariate model containing SPIR, liver disease, heart failure, the interaction between SPIR and liver disease, ACE-I use, ARB use, total bilirubin, albumin, BUN, and hospitalization number ( $\leq 2$ vs. $>2$ ). The OR for liver diseases with and without SPIR and the OR of liver disease with SPIR are compared to patients without liver disease not taking SPIR (Reference group). (B) The predicted probability (with $95 \% \mathrm{Cl}$ ) of inpatient mortality in patients with liver disease, heart failure, and neither liver disease nor heart failure while using three possible doses of SPIR for that disease state. For each disease state the average total bilirubin, albumin, and BUN were used in conjunction with the proportion of patients on ACE-I and ARB medications. Details for each disease state are in the Supporting Materials. In all groups the probability is shown for hospitalization number $\leq 2$. This demonstrates that SPIR use is associated with increased mortality in patients without liver disease.

evidence for the combination of SPIR and intestinal inflammation affecting mortality. In our rodent models, we did not observe adverse outcomes when animals were treated with SPIR alone, or when colitis was induced without SPIR. In addition, we observed a dose-dependent increased mortality in the rodent models. Clinically, we observed a dose-dependent effect of SPIR on inpatient mortality which was maintained despite adjusting for important covariates associated with SPIR usage and mortality.

Although entirely speculative, SPIR therapy in the context of epithelial barrier loss could act synergistically with the $C$. difficile toxins to increase inflammation severity and mortality. During CDI, toxin A disrupts the epithelial barrier from the apical side, potentiating toxin $\mathrm{B}$ access to receptors in the basolateral side. ${ }^{25}$ While toxin $\mathrm{B}$ is 
essential for virulence, toxin B alone does not cause symptoms unless mucosal damage has occurred. ${ }^{26-28}$ Toxin B, via a Rho/Rac signaling pathway, induces inducible nitric oxide synthase (iNOS), subsequent loss of epithelial barrier function, increased permeability, increased chloride secretion, and diarrhea. ${ }^{29}$ In our rodent colitis models SPIR had no effect in rodents with intact colons, but produced rapid mortality after damage to the intestinal epithelial barrier.

To our knowledge, SPIR has not been reported to disrupt the epithelial barrier, but SPIR does affect iNOS signaling. In the heart, SPIR induces iNOS while aldosterone represses iNOS, suggesting a functional link between the iNOS pathway and SPIR signaling. ${ }^{30}$ In contrast to SPIR, ACE inhibitors (captopril) and ARBs (candesartan, losartan) have been reported to reduce iNOS expression in the heart and kidney. ${ }^{31-33}$ We postulate that during the loss of barrier integrity caused by colonic inflammation, SPIR can act synergistically with $C$. difficile toxin B by further inducing iNOS, contributing to additional barrier disruption and increasing disease severity and mortality.

\section{CONCLUSIONS}

Clostridium difficile is a major source of hospitalacquired infections. ${ }^{34}$ Based on our results in the rodent models and the human cohort study, we propose that temporary discontinuation of SPIR in patients during CDI without liver disease could significantly reduce mortality in the more than 2.7 million hospitalized patients in the U.S. with CDI each year. ${ }^{35}$ We estimate that a similar number of patients across Europe are hospitalized with CDI. Assuming that $8.9 \%$ of CDI patients in Europe and the US are on SPIR therapy (as in our sample), a 2-fold reduction in mortality among SPIR users with CDI would save an estimated 35,000 lives annually across Europe and the U.S. Prospective clinical studies are needed to determine whether temporary discontinuation of SPIR in patients with CDI changes clinical outcomes.

\section{ACKNOWLEDGMENT}

We thank E. Rodansky of the University of Michigan for critical review of the article.

\section{REFERENCES}

1. Pitt B, Zannad F, Remme WJ, et al. The effect of spironolactone on morbidity and mortality in patients with severe heart failure. Randomized Aldactone Evaluation Study Investigators. N Engl J Med. 1999; 341:709-717.

2. Tox U, Steffen HM. Impact of inhibitors of the Renin-Angiotensinaldosterone system on liver fibrosis and portal hypertension. Curr Med Chem. 2006;13:3649-3661.

3. Mezzano SA, Ruiz-Ortega M, Egido J. Angiotensin II and renal fibrosis. Hypertension. 2001;38:635-638.

4. Rajagopalan S, Pitt B. Aldosterone as a target in congestive heart failure. Med Clin North Am. 2003;87:441-457.
5. Gullulu M, Akdag I, Kahvecioglu S, et al. Aldosterone blockage in proliferative glomerulonephritis prevents not only fibrosis, but proliferation as well. Ren Fail. 2006;28:509-514.

6. Ersoy R, Celik A, Yilmaz O, et al. The effects of irbesartan and spironolactone in prevention of peritoneal fibrosis in rats. Perit Dial Int. 2007; $27: 424-431$.

7. Nishimura H, Ito $\mathrm{Y}$, Mizuno $\mathrm{M}$, et al. Mineralocorticoid receptor blockade ameliorates peritoneal fibrosis in new rat peritonitis model. Am J Physiol Renal Physiol. 2008;294:F1084-1093.

8. Andres PG, Friedman LS. Epidemiology and the natural course of inflammatory bowel disease. Gastroenterol Clin North Am. 1999;28: 255-281, vii.

9. Sands BE, Arsenault JE, Rosen MJ, et al. Risk of early surgery for Crohn's disease: implications for early treatment strategies. Am J Gastroenterol. 2003;98:2712-2718.

10. Louis E, Collard A, Oger AF, et al. Behaviour of Crohn's disease according to the Vienna classification: changing pattern over the course of the disease. Gut. 2001;49:777-782.

11. Powell DW, Mifflin RC, Valentich JD, et al. Myofibroblasts. II. Intestinal subepithelial myofibroblasts. Am J Physiol. 1999;277: C183-201.

12. Thannickal VJ, Lee DY, White ES, et al. Myofibroblast differentiation by transforming growth factor-beta1 is dependent on cell adhesion and integrin signaling via focal adhesion kinase. J Biol Chem. 2003;278: 12384-12389.

13. Huang EH, Johnson LA, Eaton K, et al. Atorvastatin induces apoptosis in vitro and slows growth of tumor xenografts but not polyp formation in MIN mice. Dig Dis Sci. 2010;55:3086-3094.

14. Livak KJ, Schmittgen TD. Analysis of relative gene expression data using real-time quantitative PCR and the 2(-Delta Delta C(T)) method. Methods. 2001;25:402-408.

15. Kim K, Johnson LA, Jia C, et al. Noninvasive ultrasound elasticity imaging (UEI) of Crohn's disease: animal model. Ultrasound Med Biol. 2008;34:902-912.

16. Higgins PD, Johnson LA, Luther J, et al. Prior Helicobacter pylori infection ameliorates Salmonella typhimurium-induced colitis: mucosal crosstalk between stomach and distal intestine. Inflamm Bowel Dis. 2011;17:1398-1408.

17. Kamath PS, Wiesner RH, Malinchoc M, et al. A model to predict survival in patients with end-stage liver disease. Hepatology. 2001;33: 464-470.

18. Semler DE, Chengelis CP, Radzialowski FM. The effects of chronic ingestion of spironolactone on serum thyrotropin and thyroid hormones in the male rat. Toxicol Appl Pharmacol. 1989;98:263-268.

19. Charlson ME, Pompei P, Ales KL, et al. A new method of classifying prognostic comorbidity in longitudinal studies: development and validation. J Chronic Dis. 1987;40:373-383.

20. Deyo RA, Cherkin DC, Ciol MA. Adapting a clinical comorbidity index for use with ICD-9-CM administrative databases. J Clin Epidemiol. 1992;45:613-619.

21. Ochs HR, Greenblatt DJ, Bodem G, et al. Spironolactone. Am Heart J. 1978;96:389-400.

22. Overdiek HW, Hermens WA, Merkus FW. New insights into the pharmacokinetics of spironolactone. Clin Pharmacol Ther. 1985;38: 469-474.

23. Sungaila I, Bartle WR, Walker SE, et al. Spironolactone pharmacokinetics and pharmacodynamics in patients with cirrhotic ascites. Gastroenterology. 1992;102:1680-1685.

24. Patel SM, Stashefsky E, Maroun MC, et al. The impact of angiotensin-converting enzyme inhibitors and angiotensin-receptor blockers in patients with Clostridium difficile infection. Med Hypotheses. 2011; 76:813-815.

25. Carter GP, Rood JI, Lyras D. The role of toxin A and toxin B in Clostridium difficile-associated disease: past and present perspectives. Gut Microbes. 2010;1:58-64.

26. Lyras D, O'Connor JR, Howarth PM, et al. Toxin B is essential for virulence of Clostridium difficile. Nature. 2009;458:1176-1179.

27. Lyerly DM, Saum KE, MacDonald DK, et al. Effects of Clostridium difficile toxins given intragastrically to animals. Infect Immun. 1985; 47:349-352. 
28. Kuehne SA, Cartman ST, Heap JT, et al. The role of toxin A and toxin B in Clostridium difficile infection. Nature. 2010;467:711-713.

29. Witteck A, Yao Y, Fechir M, et al. Rho protein-mediated changes in the structure of the actin cytoskeleton regulate human inducible NO synthase gene expression. Exp Cell Res. 2003;287: $106-115$.

30. Chun TY, Bloem LJ, Pratt JH. Aldosterone inhibits inducible nitric oxide synthase in neonatal rat cardiomyocytes. Endocrinology. 2003; 144:1712-1717.

31. Ibrahim MA, Ashour OM, Ibrahim YF, et al. Angiotensin-converting enzyme inhibition and angiotensin AT(1)-receptor antagonism equally improve doxorubicin-induced cardiotoxicity and nephrotoxicity. Pharmacol Res. 2009;60:373-381.
32. Fan Q, Liao J, Kobayashi M, et al. Candesartan reduced advanced glycation end-products accumulation and diminished nitro-oxidative stress in type 2 diabetic KK/Ta mice. Nephrol Dial Transplant. 2004; 19:3012-3020.

33. Tian B, Liu J, Bitterman P, et al. Angiotensin II modulates nitric oxide-induced cardiac fibroblast apoptosis by activation of AKT/PKB. Am J Physiol Heart Circ Physiol. 2003;285:H1105-1112.

34. Kyne L, Hamel MB, Polavaram R, et al. Health care costs and mortality associated with nosocomial diarrhea due to Clostridium difficile. Clin Infect Dis. 2002;34:346-353.

35. Jarvis WR, Schlosser J, Jarvis AA, et al. National point prevalence of Clostridium difficile in US health care facility inpatients, 2008. Am J Infect Control. 2009;37:263-270. 